\title{
SNARE Proteins: One to Fuse and Three to Keep the Nascent Fusion Pore Open
}

\author{
Lei Shi ${ }^{1}$, Qing-Tao Shen ${ }^{2}$, Alexander Kiel ${ }^{1}$, Jing Wang ${ }^{1}$, Hong-Wei Wang ${ }^{2}$, Thomas J. \\ Melia $^{1}$, James E. Rothman ${ }^{1}$, and Frédéric Pincet ${ }^{1,3}$ \\ ${ }^{1}$ Department of Cell Biology, School of Medicine, Yale University, 333 Cedar Street, New Haven, \\ CT 06520, USA \\ ${ }^{2}$ Department of Molecular Biophysics and Biochemistry, Yale University, 333 Cedar Street, New \\ Haven, CT 06520, USA \\ ${ }^{3}$ Laboratoire de Physique Statistique, UMR CNRS 8550 associée aux Universités Paris 6 et Paris \\ 7, Ecole Normale Supérieure, 24 rue Lhomond, 75005 Paris, France
}

\begin{abstract}
Neurotransmitters are released thru nascent fusion pores, which ordinarily dilate after bilayer fusion, preventing consistent biochemical studies. Here, we employ lipid bilayer nanodiscs as fusion partners, whose rigid protein framework prevents dilation and reveals properties of the SNARE-induced fusion pore. While only one SNARE per nanodisc is required for maximum rates of bilayer fusion, efficient release of content on the physiologically-relevant time scale of synaptic transmission requires $\sim 3$ or more SNAREpins and the native VAMP2 transmembrane domain. We suggest that several SNAREpins simultaneously zippering their SNARE transmembrane helices within the freshly fused bilayers provide a radial force that prevents the nascent pore from resealing during synchronous neurotransmitter release.
\end{abstract}

Efficient synaptic transmission requires fast release of neurotransmitters from synaptic vesicles that fuse with the pre-synaptic plasma membrane upon entry of calcium ions (1). Membrane fusion necessarily implies a fusion pore that opens between the vesicle and its partner membrane at the instant of fusion. Such nascent fusion pores are typically in the range of $\sim 2 \mathrm{~nm}$ diameter based on its conductance properties, though there exists considerable variability (2-5). Neurotransmitter is released from synaptic vesicles $(\sim 40 \mathrm{~nm}$ diameter $(6,7)$ ) by diffusion through the nascent pore in the first one or two hundred microseconds, even before appreciable dilation of the pore occurs (4). The transient and variable nature of the fusion pore has severely limited biochemical and physical chemical studies.

We suggest here that nanodiscs (8-11) should provide an ideal model for such studies because the small amount of disc lipid should suffice to allow pores to open but not expand (Fig. 1 and Fig. 2A) beyond their nascent, physiologically relevant state for neurotransmitter release. Nanodiscs are synthetic lipoprotein particles that contain a small piece of circular lipid bilayer (up to $\sim 17 \mathrm{~nm}$ in diameter) wrapped by two copies of membrane scaffold protein (MSP) derived from Apolipoprotein A1. In the system we will describe here, nanodiscs contain the synaptic v-SNARE VAMP2 and small unilamellar vesicles (30-60 nm range (12)) contain the synaptic t-SNARE complex of syntaxin1 and SNAP25. SNAREs are the core machinery for this and other cellular membrane fusion processes (12-14). They 
assemble between bilayers as a four helix bundle (15) that imparts sufficient force to cause bilayer fusion (16).

After reconstitution (supplementary text), the nanodiscs containing VAMP2 (v-discs) were separated by gel filtration (Fig. 1A-C). Each disc contains about 400 lipid molecules wrapped by two MSP. With the starting ratio of MSP:VAMP2 equal to 2:6, we recovered about 7 VAMP2 per disc on average after removing VAMP2-free dics. Electron microscopy (EM) of v-discs confirmed an average diameter of $16 \pm 2 \mathrm{~nm}$ (Fig. 1D). Not surprisingly, single VAMP2 proteins on these discs are not readily distinguished because of their small size and flexible structure. However, adding the soluble t-SNARE (the complex of Syntaxin $\mathrm{H} 3$ cytosolic domain and the SNAP25N/C helical domains) forms rod-like SNARE complexes which are now seen to protrude from the nanodiscs (Fig. 1D). This confirms that VAMP2 on nanodiscs can form SNARE complexes. We used a well-established lipid mixing assay (23) to test whether v-discs can fuse with t-vesicles. NBD-

Phosphatidylethanolamine (PE) and Rhodamine-PE were included in the v-nanodiscs (1.5 mole \% each). This surface concentration effectively quenches the NBD fluorescence. However, when a nanodisc fuses with a liposome, NBD fluorescence will greatly increase because of the substantial lipid dilution (>50 fold) as disc lipid mixes with the massive excess of vesicle lipid, as we observe (Fig 2B). Little or no increase of NBD signal is observed in control experiments. The slow lipid mixing between nanodiscs and vesicles is limited by the rate of docking (initial SNARE assembly) not the rate of fusion (supplementary Fig. S1) as is the case for vesicle-vesicle fusion systems (17). A similar fusion process was observed when the SNAREs were placed in the opposite topology, with $\mathrm{v}-\mathrm{SNAREs}$ in the vesicle and t-SNAREs in the nanodisc (supplementary Fig. S2).

To monitor efflux of content via fusion pores that necessarily form at least transiently during the fusion process, calcium $(50 \mathrm{mM})$ was encapsulated in the liposomes which were then incubated with v-discs in a medium containing $2 \mu \mathrm{M}$ of a calcium - activated fluorophore, Mag-Fluo-4 (Invitrogen, $\mathrm{K}_{\mathrm{d}}$ for calcium of $22 \mu \mathrm{M}$ ). When pores open, calcium will diffuse through the pores into the exterior buffer, inducing a fluorescence signal. The results (Fig. 2C) clearly show that calcium is released in a SNARE specific manner. To ascertain that this efflux is due to diffusion thru a pore rather than from transient lysis/leakage of the vesicle during fusion, we tested the rate of release of vesicle cargos having different sizes, specifically the calcium chelator EDTA (Stokes radius: $\sim 0.4 \mathrm{~nm}$ ) and EGTA (Stokes radius: $\sim 0.5 \mathrm{~nm}$ ). EDTA was released 2.3- fold faster than EGTA liposomes (supplementary Fig. $\mathrm{S} 3$ ). From this data, a pore size of $\sim 2 \mathrm{~nm}$ can be calculated (supplementary text), similar in size to the nascent fusion pore size calculated from electrophysiological measurements (3).

When vesicles fuse to target membranes, the fusion pore eventually expands and the vesicle is incorporated into the target membrane. With nanodiscs, however, the pore cannot appreciably expand beyond its nascent diameter of $\sim 2 \mathrm{~nm}$, so the only means available to reduce membrane stress (resulting from the extreme curvature inherent to a small pore) is for the pore to eventually reseal. To confirm the prediction that nanodisc-vesicle pores reseal, we introduced dithionite $(5 \mathrm{mM})$ into samples 40 minutes after beginning the fusion assay. Dithionite will quench all externally accessible NBD (18), including NBD on both faces of unreacted nanodiscs and NBD-lipid that had diffused into the outer leaflets of liposomes via hemifusion or full fusion. Dithionite is also small enough (Stokes radius: 0.2$0.3 \mathrm{~nm}$ ) to readily diffuse through any $2 \mathrm{~nm}$ fusion pores that may remain open and quench the NBD signal on the inner leaflets in the case that the pore remains open, but will not gain access to the interior if the pore has as predicted closed off. We observe that a significant amount of the NBD dye remains protected against dithionite, and only after the fusion reaction (Fig. 2D and supplementary Fig. S4). No NBD protection was found for the negative controls. Thus, there are some fusion events that correspond to full fusion between 
the nanodisc and the liposome, in which a pore must have opened and then resealed. Reverse experiments where NBD-lipid is initially only on the t-liposomes confirm that essentially no pores remain open after full fusion (supplementary Fig. S5). Based on dithionate data, $50 \%$ of all fusion events entail full fusion with subsequent resealing of the pore (see supplementary text). The $\sim 50 \%$ balance of SNARE complex-dependent events can be accounted for by events resembling hemifusion in which no pore opens and only the outer leaflets are shared between the liposome and nanodisc. In all cases the nanodisc remains attached to the liposomes after the pore reseals (supplementary Fig. S6A-D) consistent with the idea that resealing from full fusion is to a hemifusion like state in which a stalk of lipid bilayer permanently connects the outer leaflet of the vesicle to what had been the SNARE complex containing leaflet of the nanodisc (diagrammed in supplementary Figure S6F). After resealing VAMP2 is fully resistant to toxin cleavage suggesting it is in cis - SNARE complexes (supplementary Fig. S6E, F).

The number of VAMP2 per disc can be controlled by adjusting the input VAMP2:MSP ratio. With increasing VAMP2:MSP ratios during assembly of nanodiscs, the v-disc products elute progressively earlier on gel filtration columns (Superdex-200), consistent with increasing size and more VAMP2 being inserted into each disc (Fig. 3A). To test how the number of SNAREpins affects fusion we purified seven sets of nanodiscs containing respectively an average of 1.2 (ND1), 2.2 (ND2), 3.15 (ND3), 4.3 (ND4), 5.5 (ND5), 7.4 (ND7) and 9.3 (ND9) copies of VAMP2 after VAMP2-free nanodiscs were removed by affinity purification (Fig. 3B and supplementary Fig. S7). VAMP2 and MSP were mixed in these preparations in different proportions, and the VAMP2/MSP ratio in the final isolated nanodiscs used in the fusion experiments were established by three independent methods: Coomassie Blue-based protein determinations (Fig. 3B), quantitative Western Blotting (Fig. S7) or by counting down the discrete photo-bleaching steps of single nanodisc particles containing fluorescently-labeled VAMP2 (Fig. S13). The three methods agreed very closely (within $+/-3 \%$ (SD/meanX100) for ND1 and ND2 in particular; see Table S2).

Remarkably, all seven samples drive lipid-mixing at the same rate (Fig. 3C) implying that a single v-SNARE per nanodisc yields the maximum rate of membrane fusion. Furthermore, the dithionite protection assay shows that for any VAMP2 copy number, the percentage of lipid-mixing events corresponding to full fusion remains unchanged at $45 \% \pm 7 \%$ (SD, supplementary Fig. S8). Together, these two results clearly establish that a single SNAREpin is sufficient to drive complete membrane fusion.

The pores forming in our nanodisc-liposome system are transient, eventually re-sealing of their own accord (Fig. S5). Because our lipid-mixing experiments result in full membrane fusion (i.e. full exchange of lipid) we can set a lower limit for how long these pores must have remained open. In order for all of the fluorescent phospholipid to equilibrate between the nanodisc and the liposome, the pore must remain open for $\sim 10 \mu \mathrm{s}$. (it takes $\sim 10 \mu \mathrm{s}$ for a phospholipid with a $5 \mu \mathrm{m}^{2} / \mathrm{s}$ diffusion coefficient to cover $50 \mathrm{~nm}^{2}$, half a nanodisc embedded bilayer, and reach the fusion pore). In contrast, simple considerations and in vivo observations of neurotransmitter release from synaptic vesicles suggest that a much longer time $(\sim 100 \mu \mathrm{s})$ is required for full efflux of the content the size of neurotransmitter from $40 \mathrm{~nm}$ liposomes or synaptic vesicles $(4,19-21)$.

Do the fusion pores between nanodiscs and vesicles remain open long enough for such cargo to efflux? The answer (Fig. 3D) is that they do when several or more SNARE complexes can form, but not when only a single SNAREpin is available. In contrast to the rate of lipid mixing, maximum cargo efflux decreases precipitously from 12\% (ND9 and ND7) to less than $2 \%$ (ND1 and ND2) as the number of VAMP2s per disc decreases from $\sim 9$ to $\sim 1$. Thus, even though the rate and frequency of full membrane fusion events do not depend on the 
number of VAMP2 molecules per disc, the efficiency of cargo release is highly sensitive to SNAREpin number, increasing dramatically as the number of SNAREpins increases above two per disc.

At very low SNARE numbers (i.e. ND1 or ND2) the pore opens only long enough to exchange lipid and thus only a small fraction of the content is released. Release increases starting with ND3 discs and reaches a maximum with ND7 discs -discs reconstituted with approximately 7 VAMP2 proteins total or about 3.5 per nanodisc face (Table S2) suggesting that maximum efflux requires 3 or 4 VAMP2 proteins. The simplest model is that there is a limited release when one SNAREpin is engaged and a sudden increase in content release above a threshold when enough SNAREpins are involved (Fig S12C). In the nanodisc system, that critical number is achieved when any one side of the nanodisc has at least the minimum necessary number of SNAREs. Indeed, assuming the VAMP2 distributes randomly between the two sides of each disc (Figure S12B), the calcium release across the whole of the titration fits well to such a "cooperative" model and describes the threshold number of SNAREpins for efficient content release as $~ 3$ (Figure S12C), which is consistent with in vivo observations.

The role of the SNARE transmembrane domains (TMDs) in fusion has been unclear. Membrane anchorage of the assembling cytosolic domains of VAMP and Syntaxin are needed, and when this is provided by membrane-spanning lipids fusion still occurs (22). In absolute contrast, point mutations in the Syntaxin1 TMD reduce the amplitude of the foot signal in electrophysiology (23) and deletion in the VAMP2 TMD significantly reduced neurotransmitter release (24). This implies a role for the TMDs either in the opening of the nascent physiological fusion pore, or in maintaining it open for the $\sim 100 \mu$ s needed for transmitter efflux, or both. Because fusion pores must open, at least transiently, when lipid anchors mediate fusion, the simplest possibility is that in one way or another the TMDs keep the fusion pore from resealing when transmitter is exiting and the pore has not begun to appreciably expand. In this connection, it is critical to note that the VAMP and Syntaxin TMDs extend as a two helix bundle through the entire span of the membrane (25). This raises the possibility that force resulting from the terminal zippering of SNARE TMDs within the bilayer could provide a source of energy to tip the balance against resealing in the nascent fusion pore.

To test this hypothesis, we used three chimeric VAMP2 proteins (Fig. 4A) in which the VAMP2 TMD has been replaced by (i) a dioleoyl phospholipid that just spans one monolayer (C18), (ii) a long C45 isoprenoid that can span the lipid bilayer (C45), or, (iii) a non-SNARE TMD from Platelet-Derived Growth Factor Receptor (PDGFR) (Fig. 4A). As with the wild-type VAMP2, seven samples of PDGFR-discs (characterized in supplementary Fig. S9) were prepared: PND9, PND7, PND5, PND4, PND3, PND2 and PND1 that contain respectively an average of 9.0, 7.2, 5.7, 4.4, 3.75, 2.7 and 1.2 copies of PDGFR. Lipid anchors (C18 and C45, both with an average of $\sim 5$ copies per ND) are 5 to 10 times less efficient for fusion than the VAMP2 TMD (Fig. 4B), while the PDGFR TMD fuses at the same rate as the native VAMP2 TMD at all numbers of VAMPs per nanodisc (Fig. 4C). With C18 VAMP, the fluorescence signal collapses back to the background level after dithionite injection (supplementary Fig. S10), suggesting that only the outer leaflets are shared (i.e. hemifusion). By contrast, C45 and PDGFR TMDs achieve full fusion with essentially the same $\sim 50 \%$ efficiency as the native VAMP2 TMD. This confirms that a membrane-spanning domain (be it lipid or protein) is required to achieve full fusion and also demonstrates fusion pores open when only the cytosolic domains of the SNARE complex have zippered. Thus, bilayer fusion and the concomitant opening of the nascent pore do not require assembly of the Syntaxin and VAMP TMDs into the bilayer-spanning helical bundle. 
To establish the lifetime of the open pore in these artificial fusion events, we employed the calcium release assay. By contrast to lipid mixing, none of the non-native VAMP2 TMDs efficiently released cargo (Fig. 4D, E). These experiments show that the native VAMP2 TMD is specifically required for efficient release of vesicle content after the pore opens, which it allows by virtue of lowering the rate of resealing of the nascent fusion pore.

In the nanodisc system the extent of content release can only be determined by the total amount of time the pore is open before permanently resealing. Should the pore reseal within $\sim 100 \mu \mathrm{s}$ (as calculated from diffusion constants and vesicle and pore diameter), only a commensurate fraction of the cargo will exit even though the lipids in the disc and vesicle bilayers will have fully mixed. These considerations are fundamental for understanding the different requirements for the number of SNAREs and their TMDs for vesicle fusion and content release that the nanodisc experiments reveal. Specifically, our results suggest that while a single SNARE complex suffices to open a fusion pore between nanodisc and vesicle, this pore is too short-lived to allow much transmitter to exit before the pore closes. Only when there are several or more SNAREpins assembling at the same pore does it remain open long enough for effective transmitter release. Even if there are enough SNAREs, it appears that the pore is short-lived unless the TMDs of VAMP and Syntaxin can zipper in the bilayer to keep the pore open in one way or another, perhaps by pushing outward radially as their TMDs zipper within the bilayer. A single zippering SNAREpin could not do this, explaining why it would necessarily be ineffective. But it is easy to see that three or more SNAREpins pushing away from each other radially could channel the energy of transbilayer zippering to keep the nascent pore open. In this speculative model, the restraining force against re-sealing could only last for as long as it takes the TMDs to zipper, likely to be much more than $100 \mu \mathrm{s}$ based on the maximum speed for folding of a two helix coil (2628) considering the higher viscosity of hydrocarbon as compared to water (29), ample to allow complete neurotransmitter release.

A previous study showing that a single SNARE complex could mediate vesicle-vesicle fusion also measured content mixing (30). Interestingly, correcting the published data for SNARE-free vesicles (see supplementary text) reveals that here, too, content mixing is greatly reduced relative to bilayer fusion with one SNARE complex per vesicle. Vesiclevesicle fusion is inherently a poor model of neurotransmitter release thru a nascent fusion pore because content mixing occurs not only thru a nascent pore (as in nanodisc and at the synapse) but also subsequently as the vesicles more slowly complete their fusion. Yet, despite these limitations, indications of the mechanism we have uncovered can still be found.

These findings provide a simple mechanistic basis for understanding key published data that had seemed to be contradictory. Titrations of dominant-interfering SNARE mutants in permeabilized or intact neurosecretory cells suggest a minimum requirement for three SNAREpins to open a fusion pore sufficient for neurotransmitter efflux $(31,32)$. A quantitative analysis of titrations of botulinum toxin A in relation to cleavage of SNAP25 has been interpreted to mean that a minimum of 10-15 SNAP25 molecules are required (33), but this analysis assumes that all SNAP25 is present in SNARE complexes and must be considered an upper limit only. By contrast, an elegant single particle, single molecule analysis combined with vesicle fusion reactions clearly established that a single SNAREpin was present in many fused vesicles (30). We can now see that all of these results emerge from a single underlying mechanism in which the dynamics of the nascent fusion pore are determined by the number of SNAREpins involved. Synaptic vesicles have $\sim 70$ copies of the v-SNARE VAMP2 (6) and the active zone is rich in t-SNAREs (34), ensuring that multiple SNAREpins are always available to keep the pore open and let transmitter out as rapidly as possible. 


\section{Supplementary Material}

Refer to Web version on PubMed Central for supplementary material.

\section{Acknowledgments}

This work was supported by the ANR-09-Blanc-0129 grant to Frédéric Pincet, NIH grants to James E. Rothman and a Partner University Funds exchange grant between the Yale and Ecole Normale Supérieure laboratories. We thank Dr. Jingshi Shen (University of Colorado, Boulder) for providing the PDGFR-VAMP2 expressing vector and Dr. Rainer Beck, Dr. Elizabeth Rhoades, Dr. Erdem Karatekin and Dr. Abhinav Nath for many helpful discussions. Dr. Weiming Xu and Dr. Jeff Coleman's kind help to the project was gratefully appreciated.

\section{References}

1. Sudhof TC. Annual review of neuroscience. 2004; 27:509.

2. Albillos A, et al. Nature. Oct 2.1997 389:509. [PubMed: 9333242]

3. Breckenridge LJ, Almers W. Nature. Aug-Sep;1987 328:814. [PubMed: 2442614]

4. Bruns D, Jahn R. Nature. Sep 7.1995 377:62. [PubMed: 7659162]

5. Fang Q, et al. Proc Natl Acad Sci U S A. Oct 7.2008 105:15388. [PubMed: 18829435]

6. Takamori S, et al. Cell. Nov 17.2006 127:831. [PubMed: 17110340]

7. Hu Y, Qu L, Schikorski T. Synapse. Dec.2008 62:953. [PubMed: 18798270]

8. Ritchie TK, et al. Methods Enzymol. 2009; 464:211. [PubMed: 19903557]

9. Frauenfeld J, et al. Nat Struct Mol Biol. Apr 17.2011

10. Katayama H, et al. Proc Natl Acad Sci U S A. Feb 23.2010 107:3453. [PubMed: 20142512]

11. Brewer KD, Li W, Horne BE, Rizo J. Proc Natl Acad Sci U S A. Aug 2.2011 108:12723. [PubMed: 21768342]

12. Weber T, et al. Cell. Mar 20.1998 92:759. [PubMed: 9529252]

13. Hu C, et al. Science. Jun 13.2003 300:1745. [PubMed: 12805548]

14. Sollner T, et al. Nature. Mar 25.1993 362:318. [PubMed: 8455717]

15. Sutton RB, Fasshauer D, Jahn R, Brunger AT. Nature. Sep 24.1998 395:347. [PubMed: 9759724]

16. Li F, et al. Nat Struct Mol Biol. Oct.2007 14:890. [PubMed: 17906638]

17. Smith EA, Weisshaar JC. Biophys J. May 4.100:2141. [PubMed: 21539781]

18. Xu Y, Zhang F, Su Z, McNew JA, Shin YK. Nat Struct Mol Biol. May.2005 12:417. [PubMed: 15821745]

19. Staal RG, Mosharov EV, Sulzer D. Nat Neurosci. Apr.2004 7:341. [PubMed: 14990933]

20. Pothos EN, Davila V, Sulzer D. J Neurosci. Jun 1.1998 18:4106. [PubMed: 9592091]

21. Khanin R, Parnas H, Segel L. Biophys J. Sep.1994 67:966. [PubMed: 7811953]

22. McNew JA, et al. J Cell Biol. Jul 10.2000 150:105. [PubMed: 10893260]

23. Han X, Wang CT, Bai J, Chapman ER, Jackson MB. Science. Apr 9.2004 304:289. [PubMed: 15016962]

24. Fdez E, Martinez-Salvador M, Beard M, Woodman P, Hilfiker S. J Cell Sci. Jul 15.2010 123:2473. [PubMed: 20571052]

25. Stein A, Weber G, Wahl MC, Jahn R. Nature. Jul 23.2009 460:525. [PubMed: 19571812]

26. Kubelka J, Hofrichter J, Eaton WA. Curr Opin Struct Biol. Feb.2004 14:76. [PubMed: 15102453]

27. Yang WY, Gruebele M. Nature. May 8.2003 423:193. [PubMed: 12736690]

28. Dill KA, Ozkan SB, Weikl TR, Chodera JD, Voelz VA. Curr Opin Struct Biol. Jun.2007 17:342. [PubMed: 17572080]

29. Karatekin E, Sandre O, Brochard-Wyart F. Polymer International. Apr.2003 52:486.

30. van den Bogaart G, et al. Nat Struct Mol Biol. Mar.2010 17:358. [PubMed: 20139985]

31. Hua Y, Scheller RH. Proc Natl Acad Sci U S A. Jul 3.2001 98:8065. [PubMed: 11427709]

32. Mohrmann R, de Wit H, Verhage M, Neher E, Sorensen JB. Science. Oct 22.2010 330:502.

[PubMed: 20847232] 
33. Montecucco C, Schiavo G, Pantano S. Trends Biochem Sci. Jul.2005 30:367. [PubMed: 15935678]

34. Lang T, et al. EMBO J. May 1.2001 20:2202. [PubMed: 11331586]

35. Melia TJ, et al. The Journal of cell biology. Sep 2.2002 158:929. [PubMed: 12213837]

36. Shen J, Tareste DC, Paumet F, Rothman JE, Melia TJ. Cell. Jan 12.2007 128:183. [PubMed: 17218264]

37. Heyman NS, Burt JM. Biophys J. Feb 1.2008 94:840. [PubMed: 17921206]

38. Karatekin E, et al. Proc Natl Acad Sci U S A. Feb 23.2010 107:3517. [PubMed: 20133592]

39. Permiakov, EA. Metalloproteomics. John Wiley \& Sons; Hoboken, N.J: 2009. p. xvip. 786[16] p. of plates 

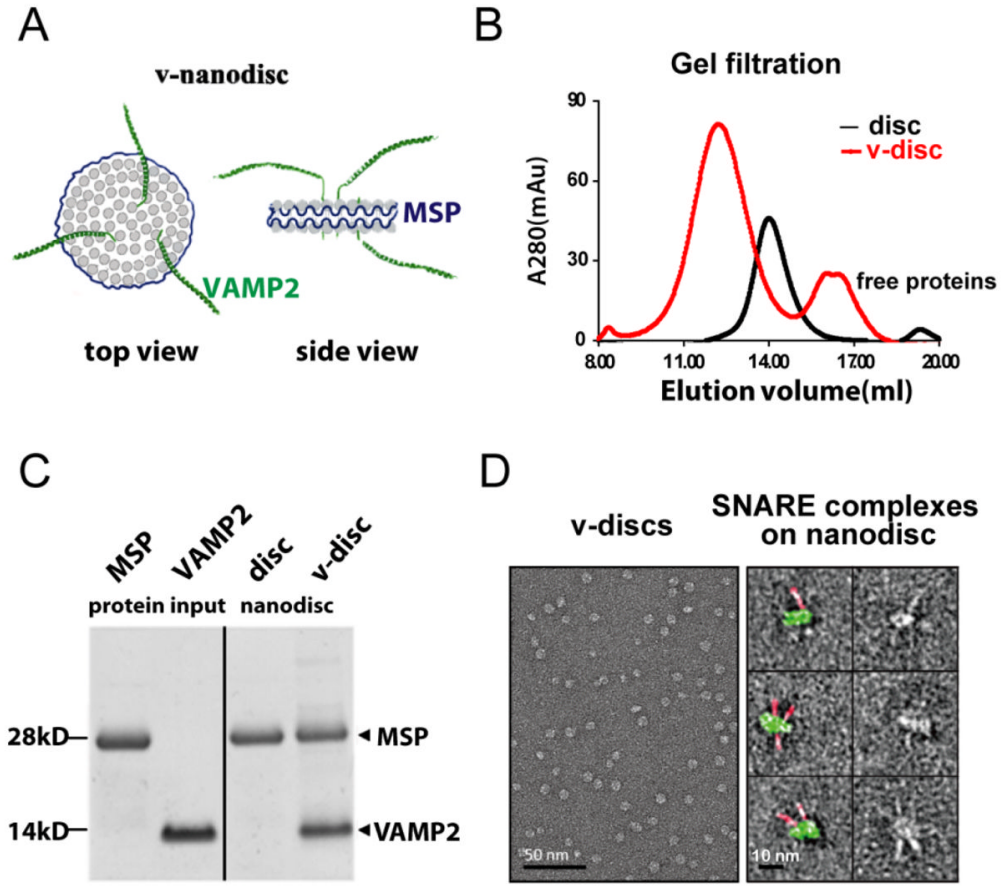

Fig 1.

(A) Cartoon showing the v-nanodisc model. The nanodisc is a small piece of lipid bilayer wrapped by two MSP (blue). VAMP2 (green) can insert into nanodisc to form V-disc (11). The lipid head groups are shown as grey spheres.

(B) Elution profile of nanodisc or v-disc on Superdex 200 10/300 GL column. Embedment of VAMP2 results in the earlier elute volume of v-disc (red major peak) as compared to that of VAMP2 free nanodisc (black peak). By gel filtration, the 6XHis-SUMO tag (cleaved from VAMP2 by SUMO-protease, the red minor peak) can also be removed.

(C) SDS-PAGE gel stained with Coomassie Brilliant Blue showing the input and final nanodisc products after gel filtration.

(D) V-disc samples were analyzed in an FEI Tecnai-12 electron microscope. V-discs showed regular "disc" shapes (Left panel), VAMP2 protein can hardly be seen because of small protein size and flexible structure. When soluble Syntaxin1A H3 domain and SNAP-25N/C domain were co-incubated with v-disc they form SNARE complexes that can be seen as rod-like structures (red, right panel) protruding from two sides of the v-disc (green, right panel). 
A

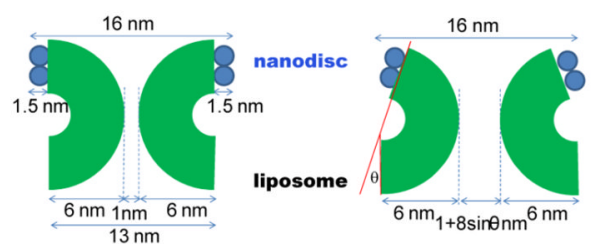

C
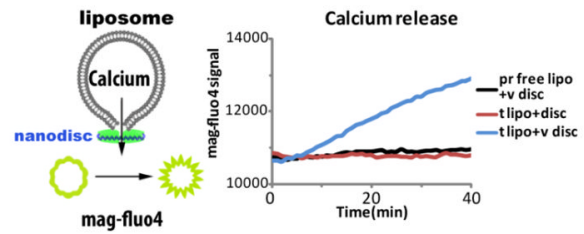

B

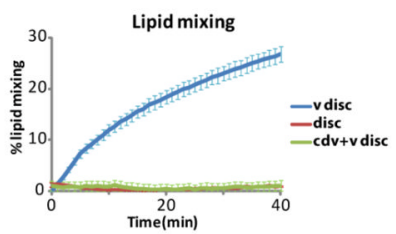

D Dithionite assay

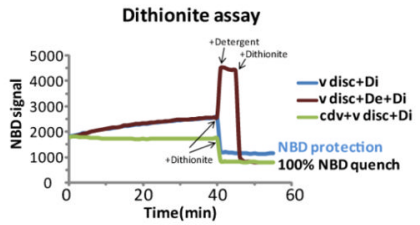

Fig 2.

(A) Schematics showing how the fusion pore can be envisioned. The diameter of the nanodisc is $16 \mathrm{~nm}$. Lipids that naturally form flat surfaces will favor structures that have a zero net curvature (when neglecting the Gaussian curvature). Hence, in saddle-like (necklike) structures, the positive (pore) and negative (perpendicular to the pore, seen in this panel) curvatures are of the same order. A $4 \mathrm{~nm}$ pore would correspond to a $6 \mathrm{~nm}$ curvature for the exterior of the bilayer. This is approximately what is represented here. With no stress a $1 \mathrm{~nm}$ pore can form. With a reasonable amount of stress $(\theta=20$ degrees $)$ a $4 \mathrm{~nm}$ pore diameter would result.

(B) Lipid mixing is SNARE specific. v-discs exchanged lipids with t-liposome (blue). Discs without VAMP2 do not fuse with t-liposomes (red), and CDV which titrates the free tSNARE also blocked the fusion (green).

(C) Calcium release is SNARE specific. $50 \mathrm{mM}$ calcium is encapsulated into t-liposome. During the liposome-nanodisc fusion, the pore opens and calcium is released from the liposome to the exterior buffer, mag-fluo-4 signal is enhanced. Increasing mag-fluo-4 signal indicates calcium is continuously released during the fusion (blue), no significant calcium release is observed under non-fusogenic conditions (red and black).

(D) Dithionite assay showed some NBD protection after 40 minutes fusion (blue). To completely quench all NBD signal, detergent (De) was first added to disrupt the liposomes followed by adding dithionite (Di) to get $100 \%$ quench (brown). With CDV to block the fusion, no NBD protection was observed after dithionite treatment (green). 
A

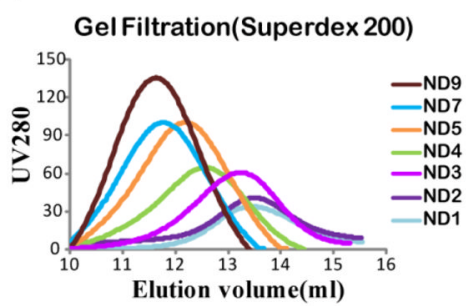

C

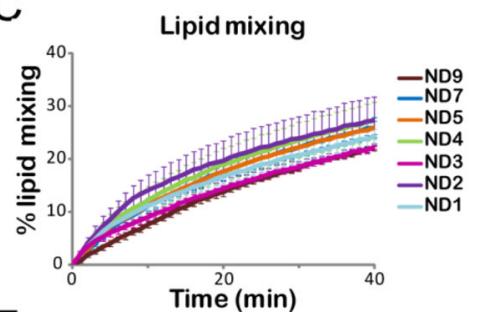

E

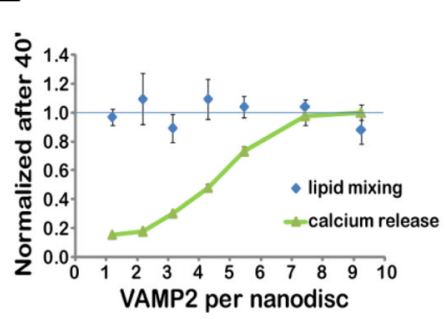

B

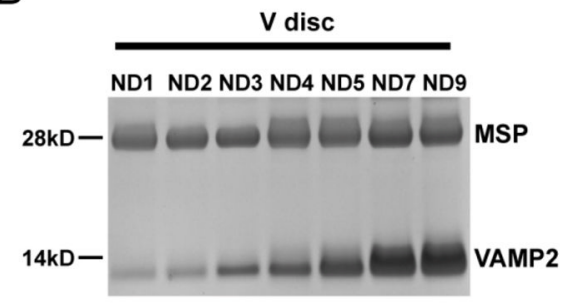

D

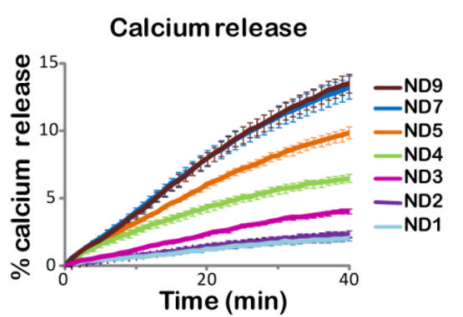

Fig 3.

(A) Elution profile of v-discs with different VAMP2 copies per disc. With more VAMP2 incorporation, v-discs eluted in smaller elution volumes on Superdex 200 column.

(B) Determination of the number of VAMP2 per nanodisc. The $\mathrm{v}$ disc samples obtained by gel filtration were analyzed by SDS-PAGE gel staining with Coomassie Brilliant Blue. The copy of VAMP2 per disc was determined by the ratio of VAMP2: MSP according to the quantification of the corresponding protein bands. Each v-disc populations have about 1.2 (ND1), 2.2 (ND2), 3.15 (ND3), 4.3 (ND4), 5.5 (ND5), 7.4 (ND7) and 9.3 (ND9) copies of VAMP2 per disc on average.

(C) Lipid mixing assays demonstrate that discs with varying VAMP2 copy numbers are equally efficient in fusing with calcium encapsulated t-liposomes.

(D) Calcium release assay showed different kinetics when $\mathrm{v}$ discs with different copy numbers of VAMP2 fuse with t calcium liposome. Calcium release is gradually increased with higher copy numbers of VAMP2 inserted into the nanodiscs.

The error bars are SEM.

(E) The end point values after $40^{\prime}$ fusion reaction are presented for both the lipid mixing (blue) and the calcium release (green). Lipid mixing, normalized by the average end point value, does not vary significantly with the number of VAMP2. Calcium release, normalized by the value for ND9, varies as a sigmoid with an inflection point at $\sim 5$ VAMP2 per nanodisc. 
A

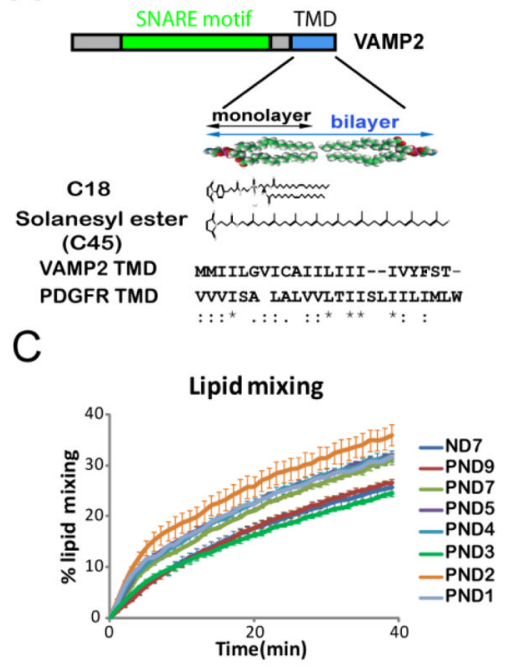

E

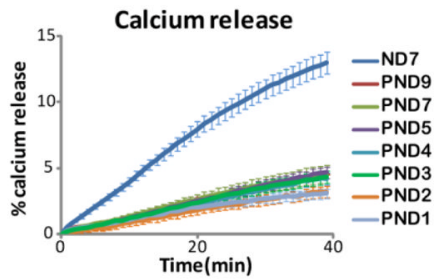

B
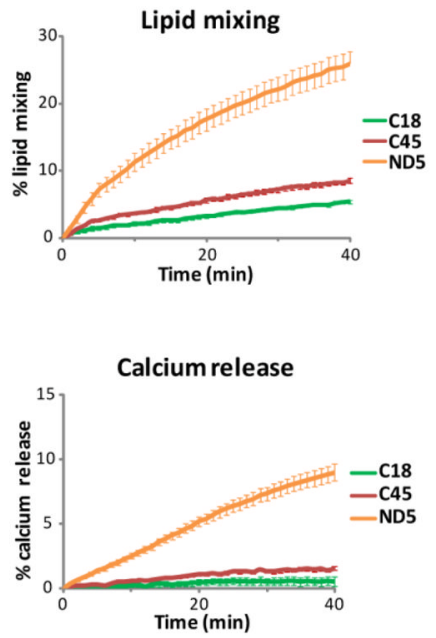

Fig 4.

(A) Schematics showing wild type and the various chimeric VAMP2. The structure of C18, C45 (solanesyl ester) and protein sequence of VAMP2 or PDGFR TMD. C18 spans only one leaflet of the lipid bilayer, while $\mathrm{C} 45$ and protein TMDs cross the lipid bilayer.

(B) Lipid mixing assay of $\mathrm{t}$ liposome with $\mathrm{v}$ disc prepared with wt VAMP2 or the CD VAMP2 with $\mathrm{C} 18$ or $\mathrm{C} 45$. The lipid anchor, either $\mathrm{C} 18$ or $\mathrm{C} 45$ showed compromised fusion efficiency comparing with wild type VAMP2 with native TMD.

(C) Lipid mixing assay showed the TMD of VAMP2 and PDGFR have similar fusion kinetics, which suggested that these two TMD are equivalent in lipid mixing.

(D) Calcium release assay demonstrated substantially reduced release when protein TMD is replaced by lipid anchor, either $\mathrm{C} 18$ or $\mathrm{C} 45$.

(E) Calcium release assay reveals VAMP2 TMD is more efficient than PDGFR TMD for content release.

The error bars are SEM. 